\title{
Ultrasonography in the Critical Care Unit
}

\author{
Keith Guevarra ${ }^{1}$ (D) Yonatan Greenstein ${ }^{1}$
}

Published online: 10 September 2020

(C) Springer Science+Business Media, LLC, part of Springer Nature 2020

\begin{abstract}
Purpose of Review This article summarizes the utility and evidence supporting the use of ultrasound exams in the intensive care unit.

Recent Findings Point-of-care ultrasonography (POCUS) is widely used by intensivists managing critically ill patients whereby they can accurately and rapidly assess for many pathologies such as pneumothorax, pulmonary edema, hydronephrosis, hemoperitoneum, and deep vein thrombosis among others. Basic and advanced critical care echocardiography, including transesophageal echocardiography, are routinely performed to determine the etiology of hemodynamic instability in undifferentiated shock and to guide subsequent therapy. The use of POCUS in the assessment of volume status is controversial with studies demonstrating that respiratory variation of the IVC is not reliable and with analysis of aortic blood flow velocity after passive leg raising maneuvers being the most promising.

Summary Point-of-care ultrasonography allows frontline clinicians to make real-time diagnoses and treatment decisions. This article will provide the reader with a broad overview of this important topic.
\end{abstract}

Keywords Critical care ultrasonography $\cdot$ Point-of-care ultrasonography $\cdot$ Intensivist $\cdot$ Echocardiography $\cdot$ POCUS

\section{Introduction}

Point-of-care ultrasonography (POCUS) has rapidly become a diagnostic tool used by frontline clinicians taking care of critically ill patients. This modality includes sonography of the heart, lungs, abdomen, kidneys, and vascular system. It incorporates diagnostic assessments and procedural guidance. It distinguishes itself from traditional ultrasonography studies in that the frontline clinician managing the critically ill patient is obtaining the images on their own and interpreting them in

This article is part of the Topical Collection on Echocardiography

Electronic supplementary material The online version of this article (https://doi.org/10.1007/s11886-020-01393-z) contains supplementary material, which is available to authorized users.

Keith Guevarra

Kpg42@njms.rutgers.edu

Yonatan Greenstein

yonatan@njms.rutgers.edu

1 Department of Medicine, Division of Pulmonary and Critical Care Medicine and Allergy and Rheumatology, Rutgers - New Jersey Medical School, University Hospital Building, Room I-354, 150 Bergen Street, Newark, NJ 07103, USA real time for immediate clinical decision making. Point-ofcare ultrasonography enables the clinician to quickly identify a multitude of life-threatening entities, to guide the management of hemodynamically unstable patients, and is standard of care for procedural guidance. Our standard practice is to perform a whole body ultrasonography study on each patient presenting with hemodynamic instability $\left[1^{*}, 2\right]$. This review will focus on the use of POCUS in the intensive care unit (ICU) and will highlight the emerging use of advanced echocardiographic techniques in addition to addressing issues of competency and credentialing.

\section{Lung Ultrasonography}

In the 1990s, Dr. Daniel Lichtenstein demonstrated the utility of lung ultrasonography in the management of critically ill patients [3,4]. This led to an international movement to define critical care ultrasonography as a basic competence for critical care physicians $[5,6]$. This movement was aptly timed with the emergence of low cost, portable ultrasonography machines. It has been shown that chest $\mathrm{x}$-ray and auscultation are insensitive in detecting pulmonary congestion in heart failure and lung ultrasonography has been shown to be 
superior in the evaluation of the dyspneic patient [7-9]. The portable chest $\mathrm{x}$-ray often has ambiguous findings, and lung ultrasonography is an invaluable tool in the intensivist's armamentarium. It can be repeated to monitor evolution of the disease process without ionizing radiation, resulting in the performance of fewer ionizing studies [10].

Video 1, clip 1, demonstrates ultrasonographic findings of the normal lung: (1) the presence of A-lines, which are reverberation artifacts that appear as regularly spaced horizontal lines separated by the same distance as that of the skin and pleural line distance, and (2) lung sliding, which is the visible dynamic movement of the visceral and parietal pleura sliding alongside each other with respiration [11]. Lung ultrasonography can identify interstitial syndrome which represents cardiogenic or non-cardiogenic pulmonary edema, consolidations, and pleural effusions, and it can diagnose and rule out pneumothoraces $\left[12^{*}\right]$.

\section{Interstitial Syndrome}

The interstitial syndrome is characterized by B-lines which are a type of comet-tail artifact (Video 1, clip 2). B-lines are hyperechoic vertical reverberation artifacts that arise from the pleural line, extend to the edge of the screen, move dynamically with respiration, and efface A-lines [3]. B-lines represent thickened interlobular septae, which may be caused by pulmonary edema, inflammation, or fibrosis. Three or more B-lines at a single interspace signifies interstitial syndrome in that interspace. The clinician must determine its etiology based on location, distribution, and clinical history.

The utility of lung ultrasonography for the diagnosis and management of cardiogenic pulmonary edema is intuitive. Animal studies demonstrate that the number of B-lines correlates strongly with the wet to dry ratio of explanted lungs [13]. The presence of B-lines diffusely in conjunction with systolic or diastolic dysfunction suggests cardiogenic pulmonary edema. In a prospective observational study of heart failure patients, the number of B-lines on admission was associated with an increased number of hospital admission days and incidence of events including the need for inotropic support [14]. Chest X-ray has been shown to lag behind the patient's clinical improvement from diuretics compared with lung ultrasonography [9]. Studies on hemodialysis patients demonstrate that the number of B-lines decreases as patients approach their dry weight [15].

\section{Pneumothorax}

Pneumothoraces occur episodically in the ICU and must always be on the differential diagnosis in mechanically ventilated patients that acutely decompensate, and in patients undergoing procedures such as central venous access. The presence of lung sliding (Video 1, clip 1) rules out the presence of pneumothorax in that area with $100 \%$ negative predictive value $[4,16,17]$. The absence of lung sliding is not diagnostic of a pneumothorax as other conditions such as prior pleurodesis, pleural adhesions, or bullous emphysema can lead to its loss. Video 1, clip 3, shows the absence of lung sliding due to a pneumothorax. The presence of "lung pulse," which is lung sliding caused by cardiophasic pulsations, indicates that the visceral and parietal pleura are fully opposed, ruling out pneumothorax. Lung pulse can be seen in patients intubated in a mainstem bronchus or in patients with a large mucus plug leading to complete atelectasis [18]. The presence of a pneumothorax can be definitively detected by locating the "lung point," the area where a fleeting appearance of lung-sliding or B-lines replaces an area of absent lung sliding during inspiration (Video 1, clip 4). This finding has $100 \%$ specificity and $66 \%$ sensitivity [19]. Our practice is to perform lung ultrasonography prior to performing invasive procedures where pneumothorax is a potential complication. The presence of lung sliding prior to the procedure and its absence post procedure is indicative of an iatrogenic pneumothorax.

\section{Pleural Effusion}

Pleural effusions are easily detected with lung ultrasonography which can provide a more detailed view of the fluid characteristics compared with computed tomography (CT). Pleural effusions may appear as an anechoic pocket of fluid (Video 1, clip 5) or as a complex pocket with cellular material or septations (Video 1 clips 6-7). Studies show that pleural effusions with echogenic material or septations within them are always exudative, while anechoic effusions may be transudative or exudative [20,21]. Unilateral pleural effusions are often found in post cardiotomy syndrome or severe mitral valve regurgitation. Ultrasonography is routinely used to identify a safe pocket for thoracentesis. Linear ultrasonography with color Doppler can be used to identify aberrant blood vessels along the needle path prior to procedure commencement [22].

\section{Consolidation}

Consolidated lung is airless and is seen with ultrasonography since no tissue-air interface exists to interfere with imaging. Lung consolidation may represent pneumonia, atelectasis, tumor, or pulmonary infarct. Integration with the clinical picture is needed to determine the etiology.

\section{Lower Extremity Deep Vein Thrombosis Studies}

Deep vein thromboses (DVTs) despite chemical thromboprophylaxis occur in approximately $12 \%$ of ICU 
patients $[23,24]$. When suspected, obtaining a timely diagnostic study is not always possible. Furthermore, acute pulmonary embolism (PE) is often on the differential for patients with hemodynamic instability, and the combined findings of a large right ventricle (RV) and a lower extremity DVT may signify the presence of an acute PE. Compression ultrasonography performed by the intensivist is quick and has a diagnostic accuracy of $95 \%$ compared with radiology performed duplex ultrasonography studies $\left[25^{\circ}, 26\right]$. A normal vein is easily compressible (Video 2, clip 1). A DVT is diagnosed when a thrombus is visualized in the vein (Video 2, clip 2) or the vein is not fully compressible (Video 3, clip 3). Intensivists commonly perform a five-point compression study of each leg: (1) common femoral vein (CFV) at the common femoral artery, (2) CFV at the greater saphenous intake, (3) CFV at the bifurcation of the common femoral artery to the superficial and deep femoral artery, (4) bifurcation of the CFV into the superficial and deep femoral vein, and (5) popliteal vein. The addition of color Doppler has not been shown to increase sensitivity [27].

\section{Abdominal and Retroperitoneal Ultrasonography}

The critically ill patient often has multiple indications for abdominal and retroperitoneal ultrasonography.

\section{Acute Kidney Injury (AKI)}

Acute kidney injury occurs in 15-38\% of critically ill patients, and rapid assessment assists in its management [28]. Video 3, clip 1, demonstrates the sonographic appearance of a normal kidney. A minority of patients have bilateral hydronephrosis from an obstructive uropathy and POCUS readily identifies this. Observation of bilateral hydronephrosis mandates assessment of the bladder for distension, which likely signifies bladder outlet obstruction. Video 3, clips 2 and 3, demonstrates hydronephrosis and a markedly distended bladder, respectively. Unilateral hydronephrosis generally requires CT imaging to further elucidate the etiology, although a skilled sonographer may identify an obstructing and infected ureteral stone prompting urgent removal $[29,30]$.

The use of bladder ultrasonography to assess urine volume qualitatively or quantitatively can help clinicians avoid the placement of indwelling foley catheters and thus reduce catheter associated urinary tract infections [31].

\section{Hypotension}

Hypotension in a critically ill patient raises the possibility of multiple abdominal processes. In trauma patients or patients that recently underwent an abdominal procedure, free fluid in the abdomen may represent hemoperitoneum. Intensivists typically apply the focused assessment with sonography for trauma examination [32] in their search for abdominal free fluid. In a non-trauma population, identified fluid may represent ascites rather than blood. Video 3, clip 4, demonstrates hemorrhagic ascites after an abdominal paracentesis, called a "hematocrit sign" [33]. The layering echogenicity suggests the accumulation of cellular material such as blood or pus. If the clinician suspects abdominal pathology, a normal abdominal ultrasound does not have sufficient sensitivity to rule out an intrabdominal catastrophe. Computed tomography or angiography should be pursued for further workup.

\section{Aortic Dissection}

Screening abdominal aortic aneurysm examinations are not routinely performed by most intensivists; however, thoracic and abdominal aortic aneurysms and dissections may be identified. Identification of an aortic aneurysm or dissection should be further evaluated by CT imaging if the patient is stable enough for the study.

\section{Basic Critical Care Echocardiography}

Basic and advanced critical care echocardiography (CCE) was first described in the 2009 statement of competence in critical care ultrasonography [3]. Consensus has been reached by the international community that basic CCE has important clinical utility [34]. This section covers the central tenets of basic CCE. The views included in a basic CCE examination are shown in Table 1. The aim of basic CCE is to answer limited questions, focused on target-oriented qualitative assessments that help guide management. The examination is often repeated the same day or on subsequent days to assess the effects of therapeutic interventions. Cognitive skills required for basic CCE include the assessment of global left ventricular (LV) size and function, identification of segmental vs. global wall motion abnormalities, right ventricular size and function, and identification of severe valvular dysfunction based on color Doppler. Basic CCE assists in the correct categorization of shock and may help identify imminent life-threatening causes of shock [35]. Basic CCE can rapidly determine if there is evidence of hypovolemic shock, a pericardial effusion, and possible cardiac tamponade. The identification of an enlarged right ventricle (RV) may suggest acute cor pulmonale and impending RV failure. We previously reported on a patient admitted with septic shock on norepinephrine presumed to be from a urinary tract infection who on POCUS was found to have severe RV dilatation and a common femoral vein DVT. The POCUS examination rapidly changed the diagnosis and management focused on thrombolytics for massive pulmonary embolism [36]. A normal basic CCE exam suggests a 
Table 1 The basic critical care echocardiogram

\begin{tabular}{ll}
\hline View & Main utility \\
\hline Parasternal long axis & Global assessment of visible structures and function \\
$\begin{array}{l}\text { Parasternal short axis (midventricular } \\
\text { level) }\end{array}$ & LV systolic function, segmental wall motion analysis, septal \\
Apical 4-chamber & RV to LV ratio \\
Subcostal long axis & Global assessment of visible structures and function \\
Inferior vena cava long axis & Assessment of volume status \\
\hline
\end{tabular}

$L V$ left ventricle, $R V$ right ventricle distributive shock state, and POCUS can be used on other organ systems to enhance diagnostic accuracy. The intensivist performing basic CCE uses this modality on critically ill, hemodynamically unstable patients, as some of the findings may not represent actionable pathology in an otherwise stable patient. For example, severe LV systolic dysfunction or RV dilatation may be chronic and may not be the primary etiology of the patient's clinical presentation. The intensivist with basic CCE capability must fully understand the shortcomings of the assumptions made when performing basic CCE.

\section{Advanced Critical Care Echocardiography}

A subset of intensivists has expertise in advanced CCE. Advanced CCE is most akin to a quantitative echocardiogram, however focusing on emergent findings and hemodynamic assessment rather than findings that contribute to the longterm management of the patient. Table 2 lists common views and measurements that are obtained with advanced CCE. This level of echocardiography allows the intensivist to quickly and accurately make complex assessments of their patient's shock state, contributing cardiac pathologies, and to guide therapy. A full review of advanced CCE is beyond the scope of this paper. A few areas are highlighted.

\section{Assessment of Cardiac Output}

The intensivist may not be concerned with the patient's left ventricular ejection fraction (EF) as patients with low EF can have normal cardiac outputs and vice versa. Rather, the intensivist focuses on the patient's cardiac output in order to categorize the patient's shock state and guide therapy. The left ventricular outflow tract (LVOT) diameter is measured via a parasternal long axis view. The LVOT velocity time integral (VTI) is measured via the apical-5-chamber or 3-chamber views.

Using the following formula, cardiac output is estimated:

Cardiac Output $=\left(\pi\left(\text { Diameter }_{\mathrm{LVOT}} / 2\right)^{2} \times \operatorname{VTI}_{\mathrm{LVOT}}\right)$

$\times$ heart rate

Table 2 The advanced critical care echocardiogram

\begin{tabular}{|c|c|}
\hline View & Points of assessment \\
\hline Parasternal long axis & Global assessment of visible structures and function; color Doppler MV and AV; EPSS; LVOT diameter \\
\hline Right ventricular inflow & Color Doppler TV, PASP estimation \\
\hline $\begin{array}{l}\text { Parasternal short axis (all } \\
\text { levels) }\end{array}$ & Segmental wall motion analysis; septal kinetics; color Doppler, CW, PW of valves, PA acceleration time \\
\hline Apical 4-chamber & $\begin{array}{l}\text { RV:LV ratio; assessment of MR and TR; PASP estimation; TAPSE; } \mathrm{S}^{\prime} \text { velocity; wall motion; diastology; pulmonary } \\
\text { venous flow analysis }\end{array}$ \\
\hline Apical 5-chamber & $\mathrm{LVOT}_{\mathrm{VTI}}$ \\
\hline Apical 3-chamber & Assessment of MR, LVOT $\mathrm{VTI}$; wall motion \\
\hline Apical 2-chamber & Assessment of MR; wall motion \\
\hline Subcostal long axis & Global assessment of visible structures and function; color Doppler MV, TV; RV:LV ratio \\
\hline $\begin{array}{l}\text { Subcostal short axis (all } \\
\text { levels) }\end{array}$ & Segmental wall motion analysis; septal kinetics; color Doppler, CW, PW of valves, PA acceleration time \\
\hline IVC longitudinal & Assessment of volume status, hepatic venous flow analysis \\
\hline
\end{tabular}

$M V$ mitral valve, $A V$ aortic valve, EPSS E-point septal separation, $L V O T$ left ventricular outflow tract, $T V$ tricuspid valve, $P A S P$ pulmonary artery systolic pressure, $C W$ continuous wave, $P W$ pulsed wave, $P A$ pulmonary artery, $R V$ right ventricle, $L V$ left ventricle, TAPSE tricuspid annular plane systolic excursion, $M R$ mitral regurgitation, $T R$ tricuspid regurgitation, $L V O T_{V T I}$ left ventricular outflow tract velocity time integral, $I V C$ inferior vena cava 
The intensivist may forgo formal calculation and instead make decisions based on the $\mathrm{VTI}_{\mathrm{LVOT}}$ alone, understanding that a normal $\mathrm{VTI}_{\mathrm{LVOT}}$ is $18-22 \mathrm{~cm}$. An average of two to three cardiac cycles should be used for patients with normal cardiac rhythms. When an irregular rhythm exists, such as in atrial fibrillation, a single LVOT VTI may not accurately reflect the true stroke volume and the clinician may need to average additional cardiac cycles. When cardiogenic shock is identified, inotropes are titrated to the VTI and clinical parameters rather than clinical parameters alone. Accurate Doppler angle is of paramount importance, and while these measures are not always feasible in critically ill patients, the existing literature supports their use [37, 38 $]$.

\section{Assessment of Valvular Pathology}

The advanced CCE intensivist is skilled with the application of pulsed wave and continuous wave Doppler for the assessment of valvular stenosis and regurgitation. Typical applications include estimating the pulmonary artery systolic pressure using the modified Bernoulli equation and estimating the aortic valve area using the continuity equation in cases of aortic stenosis. The degree of regurgitation can be assessed by jet area or the vena contracta width; however, other quantitative methods using spectral Doppler could include pressure half time, deceleration time, inflow velocity, maximal regurgitant velocity, Doppler signal intensity, and calculating of regurgitant volume and fraction to inform the final quantification [39].

\section{Assessment of Diastolic Dysfunction}

Diastolic dysfunction occurs in $20-57 \%$ of patients with sepsis [40-43] and is associated with increased mortality [44-47]. Intensivists often encounter patients with bilateral pulmonary infiltrates of unclear etiology and recognize that normal systolic function does not exclude cardiogenic pulmonary edema. The ability to assess LV diastolic function and left atrial pressure (LAP) provides real-time information that has direct implications to management. A new elevation of LAP during a spontaneous breathing trial indicates a load-related failure of the trial and may prompt the intensivist to focus on rate control, pre-load reduction, and afterload reduction [48, 49]. The intensivist may opt to extubate a patient with diastolic dysfunction and elevated LAP to non-invasive positive pressure ventilation to decrease the loss of preload and afterload reduction that occurs immediately after extubation [50]. The advanced CCE capable intensivist is familiar with the American Society of Echocardiography guidelines on diastolic dysfunction, but also considers the utility of simplifying the assessment with a reliance on the E/e' ratio [51, 52]. Further research is required in this area.

\section{Assessment of Cardiac Tamponade}

The determination of cardiac tamponade can be a complex task, often beyond the scope of basic CCE. Intensivists with advanced CCE capability incorporate spectral Doppler to assess for evidence of cardiac tamponade. Ultrasound-assisted pericardiocentesis via an apical or subxiphoid approach is easily performed by the intensivist who routinely accesses small vessels and fluid cavities throughout the body using standard Seldinger technique. Video 4 demonstrates a pericardial effusion found on a hemodynamically unstable patient. Echocardiographic tamponade was not clear; however, clinical instability raised suspicion that the effusion was clinically significant, and the intensivist accessed the pericardial space finding purulent pericarditis [53].

\section{Assessment of Right Ventricular Function and Pulmonary Hypertension}

Right ventricular dysfunction is common in the ICU [54]. Fluid loading from sepsis bundles can have deleterious effects on patients with compromised RVs. Assessment of RV function is a complex task; however, analysis of the movement of the lateral annulus of the tricuspid valve is a useful and easily repeatable measurement for the intensivist. Tricuspid annular plane systolic excursion with a normal value $\geq 16 \mathrm{~mm}$ is often used [55]. Additionally, the tissue Doppler $S^{\prime}$ wave velocity with a normal value $\geq 10 \mathrm{~cm} / \mathrm{s}$ is used [55].

Pulmonary hypertension may be encountered in the ICU. Its identification may be a sign of an acute pulmonary embolism or may indicate the need for urgent administration of pulmonary vasodilators. Using the parasternal short axis view or the subxiphoid view at the base of the heart, the pulmonary artery acceleration time (AT) is measured. Vieillard-Baron and colleagues found the AT in patients with acute cor pulmonale from ARDS and from massive PE to be $76 \mathrm{~ms} \pm$ $27 \mathrm{~ms}$ and $68 \mathrm{~ms} \pm 36 \mathrm{~ms}$, respectively. A biphasic pulmonary artery velocity envelope is also consistent with acute cor pulmonale [56].

\section{Transesophageal Echocardiography}

Transthoracic echocardiography is not feasible for a proportion of critically ill patients. Given the importance of an echocardiographic assessment in hemodynamically unstable patients, a natural approach is to incorporate transesophageal echocardiography (TEE) when transthoracic is not feasible, a position supported by the American College of Cardiology and the American Heart Association [57]. Several studies have demonstrated that trained intensivists and trained emergency physicians can safely and accurately perform critical care TEE $\left[58,59,60^{\circ}, 61-65\right]$. Some critical care physicians perform a focused TEE with four views, while others perform 
for a more comprehensive examination; this is typically dictated by the stability of the patient (Table 3 ).

\section{Assessment of Volume Status}

Patients with shock are frequently cared for by intensivists in the ICU. An eternal challenge seen daily is determining an individual's volume status and objectively assessing their likelihood to have augmentation in cardiac output with the infusion of intravascular volume. A comprehensive review of this topic is beyond the scope of this article. Using the respiratory variation of the inferior vena cava (IVC) to determine volume responsiveness was widely adopted; however, recent research shows poor sensitivity (38-55\%) and specificity (61-70\%) for this application $\left[66,67^{\circ}, 68\right]$. Many have thus deemphasized the value of this measurement. If the IVC is $<1 \mathrm{~cm}$, and the patient does not have evidence of pulmonary edema, there is a high probability of fluid responsiveness [35]. Measurement of the respiratory variation of the superior vena cava via TEE is a validated method of determining volume responsiveness. Using a cutoff of $21 \%$, this measure has sensitivity of $63 \%$ and specificity of $81 \%$ [67 $]$.

A promising method of non-invasively assessing volume status utilizes the passive leg raise maneuver, whereby a patient in a recumbent position has their legs passively raised to $30-45^{\circ}$. The intensivist obtains a baseline measurement of the stroke volume or cardiac output before the maneuver and again after the legs have been raised for 2 minutes. This method to has a sensitivity of $63-77 \%$ and a specificity of $89-$ $100 \%$ for predicting volume responsiveness [69, 70]. Despite the data to support its use, obtaining accurately repeatable measurements is challenging and the intensivist must make sure that the difference in measured velocities is from augmentation in stroke volume, not a change in Doppler angle or position [37].

\section{Training, Competence, and Credentialing}

It has been more than 10 years since most stakeholders have agreed that POCUS should be a basic competency for intensivists [3, 4]. Unfortunately, not all fellowship programs offer training, and thus, not all graduating fellows are competent in POCUS. A recent survey study of academic critical care training programs demonstrated a discrepancy between recognition of the utility and importance of POCUS and the allotment of resources and faculty time to its implementation $[71,72]$.

Intensivists have many opportunities to learn POCUS. Despite the aforementioned limitations, many graduating fellows are fully competent in POCUS. Intensivists that were not fellowship trained can acquire the skills and knowledge through local mentorship or courses which are regularly given on a local, regional, and national level. A 3-day national-level course with minimal didactics and a heavy focus on hands-on image acquisition and image interpretation has been shown to be effective for learners to rapidly accumulate a strong foundation in POCUS [73]. The American Society of Echocardiography recently published recommendations to guide existing echocardiography laboratories participating in POCUS training [74].

There is no national-level board certification for POCUS. Fellowship trained intensivists typically have their competence documented by their program director. Many nonfellowship trained clinicians have opted to pursue a certificate of completion program through CHEST (American College of Chest Physicians) to demonstrate that they have participated in a longitudinal training program which includes didactics, hands-on ultrasound scanning sessions with human models, the development of an image portfolio, and a summative written and hands-on examination. The Alliance for Physician Certification and Advancement offers a certificate program which requires the passing of a written examination and the attestation of skill by a peer evaluator. Several assessment
Table 3 Critical care transesophageal echocardiography

\begin{tabular}{ll}
\hline Standard exam views & Focused exam views \\
\hline Midesophageal AV short-axis (rotation: $30^{\circ}-45^{\circ}$ ) & Midesophageal 4-chamber (rotation: $0^{\circ}$ ) \\
Midesophageal AV long-axis (rotation: $120^{\circ}-135^{\circ}$ ) & Midesophageal AV long-axis (rotation: $120^{\circ}-135^{\circ}$ ) \\
Midesophageal bicaval (rotation: $0^{\circ}$ and $90^{\circ}$ ) & Midesophageal bicaval (rotation: $0^{\circ}$ and $90^{\circ}$ ) \\
SVC M mode (rotation: $90^{\circ}$ ) & Transgastric midpapillary short-axis (rotation: $0^{\circ}$ ) \\
Midesophageal 4-chamber (rotation: $0^{\circ}$ ) & \\
Midesophageal long-axis (rotation: $90-120^{\circ}$ ) & \\
Transgastric midpapillary short-axis (rotation: $0^{\circ}$ ) & \\
Transgastric long-axis (rotation: $120^{\circ}$ ) & \\
Midesophageal ascending aorta short-axis (rotation: $0^{\circ}$ ) & \\
Thoracic aorta (rotation $0-90^{\circ}$ ) & \\
\hline
\end{tabular}

$A V$ aortic valve, $S V C$ superior vena cava 
tools have been developed and validated and should be considered for use $[75,76]$.

Credentialing is done at the local hospital level for POCUS. Many intensivists have had success with local credentialing based on letters from fellowship program directors or completion of training courses and certificate of completion programs. At this time, programs must address credentialing individually. Setting national standards that individual institutions could use for this process would be a worthwhile endeavor.

In 2019, the National Board of Echocardiography offered the first Examination of Special Competence in Critical Care Ultrasonography. This board examination is one component towards a pathway to becoming board certified in Critical Care Echocardiography. In addition to passing this examination, the applicant must complete 150 full critical care echocardiograms that are reviewed by a qualified physician. As of the publication of this manuscript, 928 physicians have taken the examination [77]. It is expected that most academic medical centers will have at least one intensivist with this board certification in the coming years.

\section{Quality Assurance, Quality Improvement, and Billing}

A successful POCUS program must have the following components: (1) multiple ultrasound machines available for clinical use, (2) storage of captured images that meet State laws for duration, (3) generation of interpretations of performed studies with clinically relevant conclusions which are stored in the electronic health record (EHR), and (4) quality assurance with regular review of performed studies. Nascent POCUS programs often meet sharp resistance when trying to obtain permission and equipment to allow storage and the ability to generate reports in the EHR. These components are absolutely required to ensure that high-quality image acquisition and accurate interpretations are being performed. The most robust POCUS programs include interdisciplinary cooperation including radiologists and cardiologists.

Billing for POCUS studies is another domain. The ability for physicians with appropriate training to bill for and be reimbursed for ultrasound studies was established by the American Medical Association Resolution H-230.960 in 1999 and was reaffirmed in 2010 [78]. Many clinicians who routinely use POCUS choose not to bill as they see barriers to do so and find that the time it takes is not worth the reimbursement. Those that do bill find that it is a meaningful way to demonstrate the volume at which POCUS is being used. This helps to justify ultrasound related equipment and expenses to hospital administrators who generally view POCUS purely as an expense. Regardless of billing, storage of images and documentation of a report in the EHR should be standard practice.
Koenig and colleagues have published a thorough review of this topic [79].

\section{Practical Use of POCUS}

When we see a new patient with hemodynamic instability our routine is to perform whole body ultrasonography $\left[1^{\circ}, 2,35\right]$. Rounds in the ICU are conducted with the ultrasonography machine at the bedside for every patient with hemodynamic instability. We generally start with lung ultrasonography to rule out pneumothorax and assess for pulmonary edema, consolidations, and pleural effusions. We then assess the cardiac function to determine if there is a cardiac contribution to the hemodynamic instability or if there are precautions that must be undertaken given the patient's cardiac function. If the patient has renal failure, we quickly assess for hydronephrosis. Ultrasonography of the abdomen is used to assess for free fluid or other clinically indicated abdominal pathologies. Finally, a lower extremity DVT study is performed. All of this is typically achievable in under 8 minutes, and our first year critical care fellows become proficient in this modality over the first few months of their training. During the initial surge of the SARS-CoV-2 viral pandemic, our ability to seamlessly incorporate POCUS allowed our ICU to continue to deliver high quality care despite difficulty in safely obtaining routine diagnostic imaging studies, and to reduce the amount of personal protective equipment use. Over the years we have diagnosed many findings that were occult to the history and physical examination but were of critical importance to the management of the patient. For this reason, we cannot sleep at night, knowing that we provided high-quality care to our patients without performing a POCUS study on new, hemodynamically unstable, ICU admissions.

\section{Conclusions}

Point of care ultrasonography has a tremendous role in the ICU where it can be used for the initial diagnostic assessment of a hemodynamically unstable patient and for subsequent follow up of therapeutic interventions. Critical care ultrasonography has been a recommended part of critical care fellowship training for more than decade with competencies clearly delineated $[5,6]$. We strongly believe that all intensivists should incorporate POCUS into their standard of care and that hospitals should provide resources to support a successful program.

\section{Compliance with Ethical Standards}

Conflict of Interest The authors declare that they have no conflict of interest. 
Human and Animal Rights and Informed Consent This article does not contain any studies with human or animal subjects performed by any of the authors.

\section{References}

Papers of particular interest, published recently, have been highlighted as:

- Of importance

•- Of major importance

1•. Volpicelli G, Lamorte A, Tullio M, Cardinale L, Giraudo M, Stefanone V, et al. Point-of-care multiorgan ultrasonography for the evaluation of undifferentiated hypotension in the emergency department. Intensive Care Med. 2013;39:1290-8 This study shows the utility of whole body ultrasonography in a prospective cohort of 108 patients with undifferentiated shock. Studies were completed in an average of $4.9 \mathrm{~min} \pm 1.3 \mathrm{~min}$. The ultrasound diagnosis had a very high concordance with final diagnosis, $k=0.971$ (95\% CI 0.932-1.000; $p<0.0001)$.

2. Narasimhan M, Koenig SJ, Mayo PH. A whole-body approach to point of care ultrasound. Chest. 2016;150(4):772-6.

3. Lichtenstein D, Meziere G, Biderman P, Gepner A, Barre O. The comet-tail artifact. An ultrasound sign of alveolar-interstitial syndrome. Am J Respir Crit Care Med. 1997;156:1640-6.

4. Lichtenstein DA, Menu Y. A bedside ultrasound sign ruling out pneumothorax in the critically ill. Lung sliding Chest. 1995;108: 1345-8.

5. Mayo PH, Beaulieu Y, Doelken P, Feller-Kopman D, Harrod C, Kaplan A, et al. American College of Chest Physicians/La Societe de Reanimation de Langue Francaise statement on competence in critical care ultrasonography. Chest. 2009;135:1050-60.

6. Expert Round Table on Ultrasound in ICU. International expert statement on training standards for critical care ultrasonography. Intensive Care Med. 2011;37:1077-83.

7. Wang CS, FitzGerald JM, Schulzer M, Mak E, Ayas NT. Does this dyspneic patient in the emergency department have congestive heart failure? JAMA. 2005;294:1944-56.

8. Zanobetti M, Poggioni C, Pini R. Can chest ultrasonography replace standard chest radiography for evaluation of acute dyspnea in the ED? Chest. 2011;139(5):1140-7.

9. Maw AM, Hassanin A, Ho PM, McInnes MD, Moss A, JuarezColunga E, et al. Diagnostic accuracy of point-of-care lung ultrasonography and chest radiography in adults with symptoms suggestive of acute decompensated heart failure. A systematic review and meta-analysis. JAMA Netw Open. 2019;2(3):e190703.

10. Oks M, Cleven KL, Cardenas-Garcia J, Schaub JA, Koenig S, Cohen RI, et al. The effect of point-of-care ultrasonography on imaging studies in the medical ICU. A comparative study. Chest. 2014;146(6):1574-7.

11. Lichtenstein D. Lung ultrasound in the critically ill. J Med Ultrasound. 2009;17(3):125-42.

12• Koenig SJ, Narasimhan M, Mayo PH. Thoracic ultrasonography for the pulmonary specialist. Chest. 2011;140:1332-41 This is a seminal review article which covers important principles of thoracic ultrasonography. Although its title implies utility only for the pulmonary consultants, any physician managing hospital inpatients will find it to be of high value.

13. Jambrik Z, Gargani L, Adamicza A, Kaszaki J, Varga A, Forster T, et al. B-lines quantify the lung water content: a lung ultrasound versus lung gravimetry study in acute lung injury. Ultrasound Med Biol. 2010;36(12):2004-10.
14. Platz E, Campbell RT, Claggett B, Lewis EF, Groarke JD, Docherty $\mathrm{KF}$, et al. Lung ultrasound in acute heart failure: prevalence of pulmonary congestion and short- and long-term outcomes. JACC: Heart Fail. 2019;7(10):859-61.

15. Trezzi M, Torzillo D, Ceriani E, Constantino G, Caruso S, Damavandi PT, et al. Lung ultrasonography for the assessment of rapid extravascular water variation: evidence from hemodialysis patients. Intern Emerg Med. 2013;8:409-15.

16. Rowan KR, Kirkpatric AW, Liu D, Forkheim KE, Mayo JR, Nicolaou S. Traumatic pneumothorax detection with thoracic US: correlation with chest radiography and CT - initial experience. Radiology. 2002;225(1):210-4.

17. Sartori S, Tombesi P, Trevisani L, Nielsen I, Tassinari D, Abbasciano V. Accuracy of transthoracic sonography in detection of pneumothorax after sonographically guided lung biopsy: prospective comparison with chest radiography. AJR. 2007;188:3741.

18. Lichtenstein DA, Lascols N, Prin S, Meziere G. The "lung pulse": an early ultrasound sign of complete atelectasis. Intensive Care Med. 2003;29:2187-92.

19. Lichtenstein D, Meziere G, Biderman P, Gepner A. The "lung point": an ultrasound sign specific to pneumothorax. Intensive Care Med. 2000;26:1434-40.

20. Yang PC, Luh KT, Chang DB, Wu HD, Yu CJ, Kuo SH. Value of sonography in determining the nature of pleural effusion: analysis of 320 cases. AJR. 1992;159:29-33.

21. Sajadieh H, Afzali F, Sajadieh V, Sajadieh A. Ultrasound as an alternative to aspiration for determining the nature of pleural effusion, especially in older people. Ann N Y Acad Sci. 2014;1019: 585-92.

22. Sekiguchi H, Suzuki J, Daniels CE. Making paracentesis safer. A proposal for the use of bedside abdominal and vascular ultrasonography to prevent a fatal complication. Chest. 2013;143(4):1136-9.

23. Marik PE, Andrews L, Maini B. The incidence of deep venous thrombosis in ICU patients. Chest. 1997;111:661-4.

24. Malato A, Dentali F, Siragusa S, Fabbiano F, Kagoma Y, Boddi M, et al. The impact of deep vein thrombosis in critically ill patients: a meta-analysis of major clinical outcomes. Blood Transfus. 2015;13: 559-68.

25• Kory PD, Pellecchia CM, Shiloh AL, Mayo PH, DiBello C, Koenig S. Accuracy of ultrasonography performed by critical care physicians for the diagnosis of DVT. Chest. 2011;139(3):538-42 A restrospective study comparing intensivist performed compression ultrasonography for assessment of DVT to a formal technician performed and radiologist interpreted study. A total of 128 intensivist performed studies were compared with formal studies. Intensivist performed studies yielded a sensitivity of $86 \%$ and specificity of $96 \%$ with a diagnostic accuracy of 95\%. Intensivist performed studies yielded results an average of $13.8 \mathrm{~h}$ before formal study results.

26. Blaivas M, Lambert MJ, Harwood RA, Wood JP, Konicki J. Lower-extremity Doppler for deep vein thrombosis - can emergency physicians be accurate and fast? Acad Emerg Med. 2000;7:120 6.

27. Lensing AW, Doris I, McGrath FP, Cogo A, Sabine MJ, Ginsberg $\mathrm{J}$, et al. A comparison of compression ultrasound with color Doppler ultrasound for the diagnossi of symptomless postoperative deep vein thrombosis. Arch Intern Med. 1997;157:765-8.

28. Koeze J, Dieperink W, van der Horst IC, Zijlstra JG, van Meurs M. Incidence, timing and outcome of AKI in critically ill patients varies with the definition used and the addition of urine output criteria. BMC Nephrol. 2017;18:70.

29. Greenstein YY, Koenig SJ. A woman in her 60s with septic shock, abdominal pain, and a positive urinalysis. Chest. 2014;145(3):e7-9. 
30. Sidhu N, Baloch NU, Greenstein Y. What is the source? Identifying the cause of septic shock in a patient. Ann Am Thoracic Soc. 2020;17:236-9.

31. Lee YY, Tsay WL, Lou MF, Dai YT. The effectiveness of implementing a bladder ultrasound programme in neurosurgical units. J Adv Nurs. 2006;57(2):192-200.

32. Patel NY, Riherd JM. Focused assessment with sonography for trauma: methods, accuracy, and indications. Surg Clin N Am. 2011;91:195-207.

33. Subbaiah TC, Greenstein Y. A 75-year old woman with cirrhosis and shock. Chest. 2019;155(4):e87-9.

34. Via G, Hussain A, Wells M, Reardon R, ElBarbary M, Noble VE, et al. International evidence-based recommendations for focused cardiac ultrasound. J Am Soc Echocardiogr. 2014;27(7):683.e1683.e33.

35. Schmidt GA, Koenig S, Mayo PH. Shock. Ultrasound to guide diagnosis and therapy. Chest. 2012;142(4):1042-8.

36. Abbasi M, Greenstein YY, Mayo PH. Point-of-care ultrasonography for the evaluation of life-threatening hypotension. Ann Am Thorac Soc. 2016;13(12):2272-4.

37. Porter TR, Shillcutt SK, Adams MS, Desjardins G, Glas KE, Olson $\mathrm{JJ}$, et al. Guidelines for the use of echocardiography as a monitor for therapeutic intervention in adults: a report from the American Society of Echocardiography. J Am Soc Echocardiogr. 2015;28: 40-56.

38- Vignon P, Begot E, Mari A, Silva S, Chimot L, Delour P, et al. Hemodynamic assessment of patients with septic shock using transpulmonary thermodilution and critical care echocardiography. A comparative study. Chest. 2018;153(1):55-64 A prospective multicenter study, whereby patients with septic shock that required advanced hemodynamic assessment had a critical care echocardiogram and invasive transpulmonary thermodilution performed. The data were interpreted independently by two experts. Agreement between the two methods was moderate to good. The critical care echocardiogram found a potential source of thermodilution inaccuracy in $\mathbf{2 8 \%}$ of patients.

39. Zoghbi W, Adams D, Bonow R, Enriquez-Sarano M, Foster E, et al. Recommendations for noninvasive evaluation of native valvular regurgitation. A report from the American Society of Echocardiography developed in collaboration with the Society for Cardiovascular Magnetic Resonance. J Am Soc Echocardiogr. 2017;30:303-71.

40. Etchecopar-Chevreuil C, Francois B, Clavel M, Pichon N, Gastinne $\mathrm{H}$, Vignon P. Cardiac morphological and functional changes during early septic shock: a transesophageal echocardiographic study. Intensive Care Med. 2008;34:250-6.

41. Sturgess DJ, Marwick TH, Joyce C, Jenkins C, Jones M, Masci P, et al. Prediction of hospital outcome in septic shock: a prospective comparison of tissue Doppler and cardiac biomarkers. Crit Care. 2010;14:R44.

42. Sanfilippo F, Corredor C, Fletcher N, Landesberg G, Benedetto U, Foex $\mathrm{P}$, et al. Diastolic dysfunction and mortality in septic patients: a systematic review and meta-analysis. Intensive Care Med. 2015;41:1004-13.

43. Gonzalez C, Begot E, Dalmay F, Pichon N, Francois B, Fedou AL, et al. Prognostic impact of left ventricular diastolic function in patients with septic shock. Ann Intensive Care. 2016;6:36.

44. Landesberg G, Gilon D, Meroz Y, Georgieva M, Levin PH, Goodman S, et al. Diastolic dysfunction and mortality in severe sepsis and septic shock. Eur Heart J. 2020;33:895-903.

45. Brown SM, Pittman JE, Hirshberg EL, Jones JP, Lanspa MJ, Kuttler KG, et al. Diastolic dysfunction and mortality in early severe sepsis and septic shock: a prospective observational echocardiography study. Crit Ultrasound J. 2012;4:8.

46. Mourad M, Chow-Chine L, Faucher M, Sannini A, Brun JP, de Guibert JM, et al. Early diastolic dysfunction is associated with intensive care unit mortality in cancer patients presenting with septic shock. Brit J Anaesth. 2014;112(1):102-9.

47. Landesberg G, Jaffe AS, Gilon D, Levin PD, Goodman S, AbuBaih A, et al. Troponin elevation in severe sepsis and septic shock: the role of left ventricular diastolic dysfunction and right ventricular dilatation. Crit Care Med. 2014;42(4):790-800.

48. Lamia B, Maizel J, Ochagavia A, Chemla D, Osman D, Richard C, et al. Echocardiographic diagnosis of pulmonary artery occlusion pressure elevation during weaning from mechanical ventilation. Crit Care Med. 2009;37:1696-701.

49. Moschietto S, Doyen D, Grech L, Dellamonica J, Hyvernat H, Bernardin G. Transthoracic echocardiography with Doppler tissue imaging predicts weaning failure from mechanical ventilation: evolution of the left ventricle relaxation rate during a sponteaneous breathing trial is the key factor in weaning outcome. Crit Care. 2012;16:R81.

50. Michard F, Teboul JL. Using heart-lung interactions to assess fluid responsiveness during mechanical ventilation. Crit Care. 2000;4: 282-9.

51. Nagueh SF, Smiseth OA, Appleton CP, Byrd BF, Dokainish H, Edvardsen $\mathrm{T}$, et al. Recommendations for the evaluation of left ventricular diastolic function by echocardiography: an update from the American Society of Echocardiography and the European Association of Cardiovascular Imaging. J Am Soc Echocardiogr. 2016;29:277-314

52. Greenstein YY, Mayo PH. Evaluation of left ventricular diastolic function by the intensivist. Chest. 2018;153(3):723-32.

53. Greenstein YY, Khanijo S, Narasimhan M, Koenig S. A man in his 60s with circulatory collapse. Chest. 2016;149(1):e11-6.

54. Krishnan S, Schmidt GA. Acute right ventricular dysfunction. Realtime management with echocardiography. Chest. 2015;147(3): 835-46.

55. Rudski LG, Lai WW, Afilalo J, Hua L, Handschumacher MD, Chandrasekaran K, et al. Guidelines for the echocardiographic assessment of the right heart in adults: a report from the American Society of Echocardiography. Endorsed by the European Association of Echocardiography, a registered branch of the European Society of Cardiology, and the Canadian Society of Echocardiography. J Am Soc Echocardiog. 2010;23:685-713.

56. Vieillard-Baron A, Prin S, Chergui K, Dubourg O, Jardin F. EchoDoppler demonstration of acute cor pulmonale at the bedside in the medical intensive care unit. Am J Respir Crit Care Med. 2002;166: $1310-9$.

57. Quinones MA, Douglas PS, Foster E, Gorscan J, Lewis JF, Pearlman AS, et al. American College of Cardiology/American Heart Association clinical competence statement on echocardiography. Circulation. 2003;107:1068-89.

58. Benjamin E, Griffin K, Leibowitz AB, Manasia A, Oropello JM, Geffroy V, et al. Goal-directed transesophageal echocardiography performed by intensivists to assess left ventricular function: comparison with pulmonary artery catheterization. J Cardiothorac Vasc Anesth. 1998;12(1):10-5.

59. Huttemann E, Schelenz C, Kara F, Chatzinikolaou RK. The use and safety of transesophageal echocardiography in the general ICU - a minireview. Acta Anaesthesiol. 2004:48:827-36.

60 Huttemann E. Transoesophageal echocardiography in critical care. Minerva Anestesiol. 2006;72:891-913 Review paper that demonstrates that TEEs are safe in critical care patients.

61. Arntfield R, Pace J, Hewak M, Thompson D. Focused transesophageal echocardiography by emergency physicians is feasible and clinically influential: observational results from a novel ultrasound program. J Emerg Med. 2016;50(2):286-94.

62. Garcia YA, Quintero L, Singh K, Lakticova V, Iakovou A, Koenig SJ, et al. Feasibility, safety, and utility of advanced critical care transesophageal echocardiography performed by pulmonary/ critical care fellows in a medical ICU. Chest. 2017;152(4):736-41. 
63. Lau V, Priestap F, Landry Y, Ball I, Arntfield R. Diagnostic accuracy of critical care transesophageal echocardiography vs cardiology-led echocardiography in ICU patients. Chest. 2019;155(3):491-501.

64. Ramalingam G, Choi SW, Agarwal S, Kunst G, Gill R, Fletcher $\mathrm{SN}$, et al. Complications related to peri-operative transesophageal echocardiography - a one-year prospective national audit by the Association of Cardiothoracic Anaesthesia and Critical Care. 2020;75:21-6.

65. Jaidka A, Hobbs H, Koenig S, Millington SJ, Arntfield RT. Better with ultrasound. Transesophageal echocardiography. Chest. 2019;155(1):194-201.

66. Charbonneau H, Riu B, Faron M, Mari A, Kurrek MM, Ruiz J, et al. Predicting preload responsiveness using simultaneous recordings of inferior and superior vena cavae diameters. Crit Care. 2014;18:473.

67. Vignon P, Repesse X, Begot E, Leger J, Jacob C, Bouferrache, et al. Comparison of echocardiographic indices used to predict fluid responsiveness in ventilated patients. Am J Respir Crit Care Med. 2017;195(8):1022-32 A multicenter prospective study that assessed the diagnostic accuracy of dynamic parameters to predict fluid responsiveness. The study compared SVC diameter respiratory variation, IVC diameter respiratory variation, Doppler velocity in the left ventricular outflow tract, and pulse pressure variation with passive leg raise maneuver. The study found the aortic delta $V \max$ to have the best sensitivity and the delta SVC to have the best specificity in predicting fluid responsiveness.

68. Kory P. Counterpoint: should acute fluid resuscitation be guided primarily by inferior vena cava ultrasound for patients in shock? No Chest. 2017;151(3):533-6.

69. Lamia B, Ochagavia A, Monnet X, Chemla D, Richard C, Teboul JL. Echocardiographic prediction of volume responsiveness in critically ill patients with spontaneously breathing activity. Intensive Care Med. 2007;33:1125-32.

70. Maizel J, Airapetian N, Lorne E, Tribouilloy C, Massy Z, Slama M. Diagnosis of central hypovolemia by using passive leg raising. Intensive Care Med. 2007;33:1133-8.
71. Chulani S, Greenstein Y, Patrawalla A, Berman A, Guevarra K. Critical care ultrasonography use at academic training programs: a survey of critical care program directors. Chest. 2019;156(4): Supplement,A916.

72. Chulani S, Greenstein Y, Patrawalla A, Berman A, Guevarra K. Critical care ultrasonography use at academic training programs: a survey of critical care fellows. Chest. 2019;156(4):Supplement, A846.

73. Greenstein YY, Littauer R, Narasimhan M, Mayo PH, Koenig SJ. Effectiveness of a critical care ultrasonography course. Chest. 2017;151(1):34-40.

74. Kirkpatrick JN, Grimm R, Johri AM, Kimura BJ, Kort S, Labovitz AJ, et al. Recommendations for echocardiography laboratories participating in cardiac point of care cardiac ultrasound (POCUS) and critical care echocardiography training: report from the American Society of Echocardiography. J Am Soc Echocardiogr. 2020;33(4): 409-422.e4.

75. Patrawalla P, Eisen LA, Shiloh A, Shah BJ, Savenkov O, Wise W, et al. Development and validation of an assessment tool for competency in critical care ultrasound. J Grad Med Educ. 2015;7(4):56773.

76. Millington SJ, Arntfield RT, Guo RJ, Koenig S, Kory P, Noble V, et al. The assessment of competency in thoracic sonography (ACTS) scale: validation of a tool for point-of-care ultrasound. Crit Ultrasound J. 2017;9:25.

77. Mayo PH, Koenig S. Advanced critical care echocardiography certification: an update. Chest. 2020;158(1):48-9.

78. American Medical Association Res. H-230.960. Privileging for ultrasound imaging. 2010. https://policysearch.ama-assn.org/ policyfinder/detail/Ultrasound $\% 20$ imaging?uri=\%2FAMADoc $\%$ 2FHOD.xml-0-1591.xml. Accessed 6/25/20.

79. Koenig SJ, Lou BX, Moskowitz Y, Narasimhan M, Mayo PH. Ultrasound billing for intensivists. Chest. 2019;156(4):792-801.

Publisher's Note Springer Nature remains neutral with regard to jurisdictional claims in published maps and institutional affiliations. 